\title{
La transversalización de la clínica jurídica como estrategia de formación en el Programa de Derecho de la Universidad Autónoma de Bucaramanga (Unab)*
}

Julián Eduardo Prada Uribe ${ }^{a}$

\begin{abstract}
Resumen: pese al reconocimiento de la importancia emancipadora de la educación, subsiste un modelo sustentado en las promesas que legitimaron el privilegio epistemológico en el siglo xIx, que han reproducido estereotipos de subordinación y minimizado las responsabilidades que el saber trae consigo. Con fundamento en lo anterior, producto del proyecto "La clínica jurídica como estrategia de formación en el Programa de Derecho de la Unab", avalado por esta institución en la convocatoria 2019-2020, y que se derivó de la intervención realizada por el autor en la Maestría en Educación en Derechos Humanos del Crefal; aquí se sistematiza una experiencia pedagógica con miras a redimensionar los desequilibrios existentes en la formación jurídica, buscando hacer del aprendizaje una actividad significativa, colaborativa, sensible y servicial.
\end{abstract}

Palabras clave: clínica jurídica; construcción de paz; educación en derechos humanos

Recibido: 20 de octubre de 2021 Aceptado: 04 de junio de 2021

Disponible en línea: 31 de diciembre 2021

Cómo citar: Prada Uribe, J. E. (2021). La transversalización de la clínica jurídica como estrategia de formación en el Programa de Derecho de la Universidad Autónoma de Bucaramanga (Unab). Prolegómenos, 24(48), 23-39. https://doi.org/10.18359/prole.5368

* Artículo de investigación Producto del proyecto: La clínica jurídica como estrategia de formación en el programa de Derecho de la UNAB, avalado por esta institución en la convocatoria 2019-2020, y que se derivó de la intervención realizada por el autor en la Maestría en Educación en DDHH del CREFAL.

a Magister en educación en Derechos Humanos. Docente Universidad Autónoma de Bucaramanga, Bucaramanga, Colombia.

Correo electrónico: jprada2@unab.edu.co ORCID: https://orcid.org/0000-0001-5737-8440 


\title{
Mainstreaming Legal Clinic as a Training Strategy in the Law Program of the Universidad Autónoma de Bucaramanga (Unab)
}

\begin{abstract}
: despite recognizing the emancipatory importance of education, a model based on the premises that legitimized epistemological privilege in the 19th century subsists, reproducing subordination stereotypes and minimizing the responsibilities that knowledge entails. Thus, as a result of the project "Legal Clinic as a Training Strategy in the Unab Law Program," endorsed by this university in the 2019-2020 call and derived from the author's intervention during his Master's in Human Rights Education at Crefal, this paper systematizes a pedagogical experience to re-gauge existing imbalances in legal training, seeking to make learning meaningful, collaborative, sensitive, and helpful.
\end{abstract}

Keywords: legal clinic; peacebuilding; human rights education

\section{A transversalização da clínica jurídica como estratégia de formação no Programa de Direito da Universidad Autónoma de Bucaramanga (Unab)}

Resumo: Apesar do reconhecimento da importância emancipatória da educação, permanece um modelo baseado nas promessas que legitimaram o privilégio epistemológico no século XIX, que reproduziram estereótipos de subordinação e minimizaram as responsabilidades que o conhecimento traz consigo. Com base nisso, como produto do projeto "A clínica jurídica como estratégia de formação no Programa de Direito da Unab", endossado pela instituição no edital 2019-2020, e que foi derivado da intervenção realizada pelo autor no Mestrado em Educação de Direitos Humanos do Crefal, neste trabalho uma experiência pedagógica é sistematizada com o objetivo de redimensionar os desequilíbrios existentes na formação jurídica, buscando tornar o aprendizado uma atividade significativa, colaborativa, sensível e útil.

Palavras-chave: clínica jurídica; construção da paz; educação em direitos humanos 


\section{Introducción}

De acuerdo con la Constitución Política de 1991, Colombia se ha erigido como un Estado Social de Derecho, fundado en la defensa de la dignidad, cuyo principal propósito es servir a una comunidad plural, promover el desarrollo sostenible y asegurar la efectividad de los derechos humanos; preservando la justicia y la participación civil en las decisiones políticas, económicas y culturales. En un entorno con tales características, la educación constituye un requisito sustancial, dispuesto a edificar valores, actitudes y comportamientos, y erigirse como un factor de progreso (Red-desc, 1999). Pese al reconocimiento de su importancia, subsiste un modelo educativo sustentado en las promesas que legitimaron el privilegio epistemológico del conocimiento en el siglo xix, que no solo no se cumplieron, sino que han reproducido estereotipos de sumisión y dependencia, han minimizado las responsabilidades que el saber conlleva y han originado una condición de vulnerabilidad sobre ciertos sectores (de Sousa, 2004).

En el ámbito jurídico latinoamericano, heredero de la civil law, los contenidos escolares se han estacionado en aspectos normativos, primordialmente legales, relativos al reconocimiento de instituciones y a la definición de reglas de validez y técnicas de interpretación. Se trata de una versión académica que se desarrolló "en Francia, a través de la escuela de la exégesis; en Alemania, a través de la jurisprudencia de conceptos; y en Inglaterra, a través de la jurisprudencia analítica" (Vázquez, 2006, p. 2); y que ha edificado una ciencia o logos dominante de corte formal, transmitida por terceros, que ya no hacen parte del contexto geográfico o pedagógico, donde se aplica, y que seguramente no representa a los sujetos que se educan en el escenario nacional, pues pretende observarlos como una especie de eruditos, en cierta medida insensibles y poco capacitados para vincular la materialización de la justicia entre las funciones del derecho. Con ello, se generó una fuerte desconfianza por parte de la comunidad (Gómez-Araújo, 2011; Taelli-Gómez, 2014).

Este enfoque quizá encarna "el principal escollo para la innovación en los planes de estudio, los métodos de enseñanza, la modificación de los perfiles de egreso y la profesión académica" (Valle-Acevedo, 2006, p. 85). No en vano, siguiendo a González-Morales (2004), León et al. (2016), Vásquez-Santa María (2016) y Merlín (2016), el modelo vigente se encuentra reducido a una visión teórica, bajo la hegemonía de la cátedra magistral y las herramientas memorísticas, sin una pedagogía vivencial y con una ineficiente formación axiológica.

En este orden de ideas, los estudiantes suelen desconocer la puesta en práctica de las destrezas adquiridas. Por eso, no sienten urgencia por dar significado al conocimiento; mientras que la actividad docente, habitualmente, se concentra en la transmisión de términos, categorías o discursos, simplificando la evaluación a la reproducción de información y configurando una fuerte devoción por el saber, proveniente de las fuentes primarias del derecho.

El nexo entre todas las características anteriores ha orientado una clase particular de egresado: ritualista, lógico y descriptivista. Al punto de que Courtis (2007), Rodríguez (2007), FigueroaCamacho (2011) y Bayuelo (2015) han advertido que, si la educación jurídica continúa fijando su objeto de estudio en un saber estático y documental, terminará obstruyendo las habilidades que el mundo exige de los abogados, para atender y resolver los problemas cotidianos.

Fue así como la Universidad Autónoma de Bucaramanga (Unab), desde hace un par de años, empezó a inquietarse por las sensaciones negativas que notó entre algunos estudiantes, advirtiendo el carácter cortoplacista que implicaba el modelo clásico de formación, por lo que inició la búsqueda de alternativas para lograr una proyección profesional más crítica y significativa. Así pues, en 2014 instituyó la Clínica Jurídica de Interés Público y Derechos Humanos (Clínica Jurídica de la Unab), como un centro académico-administrativo vinculado al departamento de práctica de la Facultad de Ciencias Jurídicas y Políticas, cuyo objeto fue definido en el sentido de

coadyuvar a la prestación de servicios de asistencia en el campo del derecho [...], convirtiéndose en un espacio de reflexión y análisis de los problemas de la comunidad, para consolidar competencias 
investigativas y de litigio. (Unab. Junta Directiva, 2014, p. 2)

Pese a los avances obtenidos, tal unidad ha abarcado esencialmente el ejercicio voluntario de los estudiantes de último año (noveno y décimo semestre). Así que el plan fruto de la experiencia que se sistematiza en este documento pretendió sumar otros espacios y niveles del programa.

Motivados por la metodología de Rancière (1996), en lugar de polemizar por qué continuar atados al sistema vigente, el punto de partida del proyecto radicó en cuestionar las condiciones en que no era dable permanecer en dicho orden y, en consecuencia, determinar la manera de llevar a cabo la emancipación. El esclarecimiento de tales desacuerdos permitió concentrar esfuerzos sobre los protagonistas del proceso: estudiantes y profesores ${ }^{1}$. A partir de esta labor, se dirigió el objetivo de la intervención hacia la subversión del orden dado, pero no con una lógica de caducidad o indisciplina, sino esperanzados por encontrar una brecha que apoyara la ruptura de las cargas impuestas, a fin de potenciar los saberes y extenderlos a quienes viven sin ser o sentirse parte de la sociedad.

1 Durante la realización del proyecto, adquirieron valor las conductas, los sentimientos y las emociones de los estudiantes, así como los estímulos de los profesores en el cumplimiento de sus metas laborales y vocacionales. Tras aplicar una encuesta exploratoria a cuarenta y cuatro estudiantes de primer año y realizar dos grupos de discusión, uno con diez estudiantes escogidos al azar, inscritos en distintos cursos de los cuatro últimos semestres de carrera; y otro con diez profesores de tiempo completo y medio tiempo; fue posible comprobar, por una parte, que, aunque los estudiantes esperan un modelo educativo apegado a la tradición, suelen preocuparse porque no recuerdan los contenidos tratados en las clases o creen que tienen poca utilidad; y por otra, que los profesores ven su práctica como una rutina, pero no siempre se sienten incómodos con ello.
Convencidos de que el modelo clínico jurídico ${ }^{2}$ cuenta con los elementos requeridos para resolver los problemas de la educación en derecho, ligados con el escaso contacto con la realidad y una inconveniente organización jerárquica; la propuesta de investigación radicó en comprobar, entre los estudiantes de primer semestre del Programa de Derecho de la Unab, por un lado, cuál era la percepción resultante tras la aplicación de la metodología, en términos de empoderamiento como personas reflexivas, creativas e innovadoras; por otro, qué impacto era capaz de ocasionar simultáneamente en la población, con respecto a la satisfacción de sus intereses o demandas.

A pesar de las diferencias con el paradigma vigente, este esquema no se separó de las directrices institucionales ni de la reglamentación nacional en torno a la oferta de programas profesionales. Por el contrario, actualizó el lazo con las obligaciones derivadas de la Declaración Universal de los Derechos Humanos (1948), el Pacto Internacional de los Derechos Económicos, Sociales y Culturales (1966), la Constitución Política de Colombia (1991) y la Ley de Educación Superior [Ley 30 de 1992]. No en vano, sirvió para fortificar el desarrollo de potencialidades humanas de manera integral, la articulación de personas y grupos dentro de la Universidad, el relacionamiento con la comunidad y las actividades de extensión.

\section{Materiales y métodos}

En el aprendizaje clínico yace una fórmula efectiva para la educación que, habiéndose inspirado en los procesos para la formación en medicina, cuenta con lo necesario para redimensionar la disparidad entre lo teórico y lo práctico, mediante herramientas de diagnóstico, prescripción e intervención alrededor de problemas reales (Bregaglio et al.,

2 El modelo clínico jurídico se atribuye al profesor Jerome Frank, quien, desde 1933, expuso las falencias del sistema vigente y justificó la necesidad de superar la visión enciclopedista del mundo del derecho, mediante una dinámica sustentada en el aprendizaje investigativo, colaborativo, experimental y protagónico. 
2013; Londoño-Toro, 2015; Vásquez-Santa María, 2016; Téllez et al., 2016). Se trata, por tanto, de una oportunidad para renovar los sistemas clásicos de enseñanza jurídica, haciendo prevalecer los principios y valores sobre la didáctica de las reglas ${ }^{3}$ y previendo que las actividades académicas no pueden continuar conduciéndose como un ejercicio de sumisión y conformismo.

Ahora bien, la descentralización y transversalización del modelo clínico jurídico en la Unab, entendido como una estrategia de mejoramiento dentro del sistema educativo formal y enfocado en el grupo de estudiantes inscritos en el primer periodo lectivo de 2019, en el curso de Diacrónica Jurídica, encarnó un movimiento de liberación y reivindicación del capital humano y de sinergia con el plano institucional, evocando un proceso horizontal y coordinado, en el que cada persona desempeñó un rol protagónico. Ello supuso incorporar en el programa de estudio de una asignatura percibida como poco útil, el desarrollo de competencias profesionales y la inclusión de factores emocionales como elementos de análisis, acción y evaluación.

Después de recrear una prueba piloto con catorce estudiantes en el segundo semestre de 2018, el primer desafío se asumió durante el periodo intersemestral y consistió en preparar la guía de clase y el cronograma de dieciséis semanas, correspondientes al calendario académico ordinario. Así, para introducir gradualmente la metodología, en las primeras sesiones se resolvió transitar desde

3 En los países de tradición romana suelen primar los códigos y otras manifestaciones legislativas en el sistema de fuentes jurídicas. Conforme con dicha herencia, las normas han adquirido, en esencia, la estructura de reglas y, por tal motivo, suelen estar expresadas en términos condicionales (causa-efecto) o correlacionales (caso-solución). Las teorías contemporáneas, a su turno, han introducido otros esquemas normativos, entre ellos, los principios y valores jurídicos, esto es, mandatos superiores del ordenamiento que reflejan una determinada visión o máximas conductuales que permiten la sistematización del ordenamiento, respectivamente (Atienza y Ruiz, 1991). una cátedra participativa ${ }^{4}$ hasta un aula invertida ${ }^{5}$, conforme con la cual los estudiantes pudieron despojarse de prevenciones y prejuicios. En este periodo, justamente, se consiguió acreditar la fuerza y la importancia que cada miembro involucraba para el curso: interpelando, rescatando y destacando sus aportes ${ }^{6}$. Se trató de una pedagogía alterada por la presencia de las diferencias, en la que los estudiantes asumieron un rol principal, donde la función docente se arraigó en acompañarlos, mediar en sus discusiones, comprometerlos y, sobre todo, confiar en sus iniciativas. Tal dinámica implicó, de igual forma, la utilización de recursos conversacionales, soportados en la presentación de dilemas, controversias y casos difíciles y trágicos, desplegando hábitos de argumentación, eficaces

4 La cátedra participativa es una propuesta concebida para animar la reflexión de los estudiantes a partir de un trabajo de problematización, sustentada en el valor de sus intervenciones y en el protagonismo alcanzado (Borrero, 2014).

5 El aula invertida permuta los dos instantes que implican la educación tradicional: la exposición de contenidos por parte del profesor y la realización de actividades por fuera del aula de clase. De este modo, las unidades temáticas son abordadas previamente y de manera autónoma por los estudiantes, mientras que la práctica y ejecución de proyectos se materializa en el salón, lo que conlleva a un alto procesamiento cognitivo, donde los estudiantes tienen que resolver problemas, pensar en aplicaciones, desarrollar habilidades blandas y comprender la complejidad de las instituciones o categorías propuestas (Merla y Yáñez, 2016).

6 Para incentivar la participación, se llevaron a cabo ejercicios con miras a controlar los actos interiores y exteriores de censura. No en vano, en palabras de Raudsepp (1963), “Nothing can inhibit a stifle the creative process more [...] than critical judgment applied to the emerging idea at the beginning stages of the creative process" (p. 211). 
para articular lo hermenéutico con lo crítico y lo comunicativo 7 .

Lo anterior, desde el punto de vista logístico, comportó el diseño de actividades curriculares y extracurriculares, pues el modelo clínico jurídico no concibe que el acto educativo se cumpla exclusivamente durante el tiempo de contacto profesor-estudiantes, sino también en otras instancias y contextos, formales e informales. Por tanto, el programa se trazó alrededor de problemas y preguntas, y no de temas o proposiciones categóricas. Además, implementó una serie de estrategias e instrumentos de evaluación para medir progresivamente las competencias de los estudiantes y no solo su aptitud para recordar determinados datos.

Paralelamente, se produjo una presentación audiovisual que fuera interactiva y provocadora, basada en infografías y fuentes alternas de conocimiento. A ello se suman dos protocolos de trabajo, uno que permaneció abierto para toda la comunidad institucional: "Cine-diálogo"; y otro específico para los estudiantes del curso, con el propósito de formular un proyecto de intervención, que serviría para consumar el paso de lo doctrinal a lo

7 Según los teóricos de la argumentación jurídica, en un ordenamiento es factible identificar casos fáciles, casos difíciles y casos trágicos. En el primer supuesto, se trata de asuntos que proveen una respuesta o solución mediante la aplicación de un juicio lógico formal (Vázquez-Sánchez, 2006); en el segundo, la cuestión exige mecanismos de ponderación y criterios de proporcionalidad u otros razonamientos morales que conduzcan a una salida justa (Atienza, 1997); y en la tercera situación, debido a la colisión de derechos fundamentales de un mismo rango, cuyo desenlace conlleva el sacrificio de uno de ellos, ante la inexistencia de una respuesta correcta, la razón no es suficiente para justificar una decisión, lo que da paso al uso de los sentimientos (Lariguet, 2004). experiencial, apoyados en herramientas de creative solving problems ${ }^{8}$.

Respaldados en sesiones presenciales de dos horas semanales, se privilegió el autoconocimiento y reconocimiento de los integrantes del grupo, la explicitación de los resultados esperados en el curso, las características del modelo de aprendizaje $y$, aprovechando experiencias previas externas e internas, se ajustaron las fases de conformidad con la propuesta de Castro-Buitrago (2015), incluidas en Enseñanza clínica del derecho. Metodologías, experiencias innovadoras y buenas prácticas.

Así, el plan estuvo encauzado a (1) situar la realidad de la clase en el contexto específico de la historia del derecho, en virtud de su significación y para asumir una posición reflexiva; (2) examinar vivencialmente los contenidos, con disposición hacia su apertura radical, es decir, desnaturalizando dogmas, resistiéndose a las verdades instaladas y cuestionándose cómo podría transformarse la realidad; (3) generar un vínculo sensible, democratizador e inclusivo con todos los sujetos parte del proyecto, tanto directa como indirectamente, para humanizar la actividad educativa; (4) asumir desde la acción el compromiso ético y político que conlleva la afirmación de la ciudadanía y la educación jurídica; y (5) evaluar el proceso cumplido, en la medida en que haya favorecido (o no) la consecución de cambios personales, institucionales y sociales.

En el trascurso del semestre, según fueron fijándose los objetivos de intervención alrededor de cinco líneas de trabajo definidas: personas con discapacidad, habitantes de la calle, víctimas del delito de trata, discriminación por razones de sexo, género u orientación sexual, y población migrante; se crearon, libremente, equipos dentro del salón, aceptando responsabilidades individuales y

8 Creative problem solving (csp) es un método para afrontar dificultades y resolver preguntas o retos, a problemas cuyas soluciones no resultan obvias, por medio de la creatividad y la innovación. Se trata de un entorno diseñado por Alex Osborn y Sidney Parnes, conveniente para redefinir y clarificar hechos, generar ideas por fuera de las estructuras tradicionales de pensamiento, desarrollar planes de acción y llevarlos a cabo (MacLeod, 1963). 
el liderazgo colectivo ${ }^{9}$. Entretanto, la labor docente supuso coadyuvar como par, desempeñando tareas equivalentes a las de los estudiantes, moderar salidas de cara a conflictos eventuales y, en términos generales, observar la dinámica y sus resultados.

\section{La clínica jurídica como estrategia de aprendizaje}

La clínica jurídica constituye una estrategia enfocada en la formación integral y el aprendizaje activo y constructivo. Su nombre se atribuye a Jerome Frank, a raíz de su ensayo publicado en 1933 Why not a clinical lawyer-school? No obstante, hasta 1960 no se entabló una discusión juiciosa acerca del tema y solo a puertas del siglo xxi el mundo hispanoamericano recogió las técnicas enunciadas.

El estado del arte en esta última región ha ido componiéndose a partir de cuatro instancias: (1) fundamentación pedagógica de la clínica jurídica; (2) reseñas generales sobre el desarrollo de la práctica clínica; (3) exploraciones y conclusiones desde la experiencia; y (4) oportunidades de aplicación o de mejoramiento metodológico.

En relación con el primer propósito, Abramovich (1999) abrió la discusión en La enseñanza del derecho en las clínicas legales de interés público, señalando su importancia como espacio de docencia, destinado a la práctica profesional y mecanismo para garantizar la vigencia de los derechos humanos y el acceso a la justicia; Blázquez-Martín (2006a), en "Apuntes acerca de la educación jurídica clínica", expuso el modelo y sus similitudes con las habilidades y competencias demandadas en el ámbito de la abogacía; de igual forma, Correa

9 La formación clínica jurídica puede caracterizarse de diversas maneras y según distintos criterios. Desde el punto de vista del área de trabajo, una clínica puede especializarse en una rama del conocimiento, puede seleccionar ciertas problemáticas sociales o fijarse en determinados grupos en situación de vulnerabilidad. En el caso de la clínica jurídica de la Unab, con el propósito de recuperar como sujetos de derechos a personas y poblaciones excluidas, se ha optado desde su origen por este último precepto. y Vásquez (2008), en "Enseñanza clínica del derecho", se dieron a la tarea de justificar la naturaleza revolucionaria del modelo clínico, con todo y la deconstrucción de los cánones clásicos de la educación jurídica, discutiendo los esquemas pedagógicos establecidos y sus concepciones conservadoras.

Por su parte, Estrada (2015) en "De las aulas a la calle: un paso más de la racionalidad teórica hacia la racionalidad práctica", resaltó el valor del aprendizaje clínico como medio para la promoción de capacidades críticas y propositivas, para el fortalecimiento de un nuevo estilo de razonamiento jurídico y para la construcción de una teoría jurídica acorde con el Estado social y constitucional de derecho. En "Perspectivas de la enseñanza clínica del derecho en Colombia", Castro-Buitrago (2015) describió las falencias de la enseñanza del derecho, sustentadas en una visión formalista y positivista, y dejó sentada su opinión sobre la actividad de los consultorios jurídicos y el surgimiento del método clínico como alternativa de educación. Por último, Martocci (2017), en el ensayo "Clínica jurídica en derechos humanos, otra pedagogía", mostró que, alzadas contra un paradigma dogmático, positivista, vertical y acrítico, las clínicas ofrecen un esquema diverso: un espacio de interrogación y comprensión del rol del derecho respecto de realidades sociales, económicas y políticas en las que continuamente se vulneran los derechos de las personas.

En lo concerniente a la recuperación y resumen de las fuentes acerca de la educación clínica, González-Morales (2004) publicó "El trabajo clínico en materia de derechos humanos e interés público en América Latina", donde aclara los progresos de este movimiento pedagógico en relación con la defensa de los derechos humanos y las acciones de interés público, insistiendo en el papel de los estudiantes y el valor de una educación sensible. A su vez, Blázquez-Martín (2006b), en "La educación jurídica clínica en el contexto del proceso de Bologna", narró cómo nació y han ido produciéndose cambios en la educación jurídica, a través de la metodología clínica, llevándolo a proponer dicha herramienta como recurso para la modernización de los planes de estudio en toda Europa.

Carrillo y Espejo (2013), con la intención de descubrir las posibilidades de obtención de justicia 
por medio de la educación, repasaron algunas de las experiencias latinoamericanas y las contrastaron con las tendencias previstas en las facultades estadounidenses, en "Re-imaginando la clínica jurídica de derechos humanos". Por último, García (2015), en "La evolución de la educación jurídica clínica en España”, destacó los atributos de transformación y renovación que, en torno a la realidad y las universidades, ha tenido este modelo desde inicios del siglo xxi.

Referidos al tercer objetivo, Castro-Buitrago (2006a), en el "Desarrollo de un método de selección de casos de interés público", se centró en el ejercicio de la Clínica Jurídica de la Universidad de Medellín y su sistema de selección de casos, como recurso pedagógico. Hurtado-Castrillón (2011), de igual forma, en la ponencia "Clínica jurídica en acciones de interés público en materia de discapacidad para el eje cafetero", describió cómo se ha cumplido esta práctica en la Clínica de la Universidad Libre de Pereira. Molina-Saldarriaga (2012), en "La enseñanza clínica del derecho", examinó la importancia de la interdisciplinariedad en la educación superior y compartió algunos de los resultados de la Clínica Jurídica de la Universidad Pontificia Bolivariana sobre cuestiones ambientales.

A los anteriores, se suma Borràs (2012), con su trabajo "La clínica jurídica de la Universitat de València: el compromís social de l'aprenentatge participatiu", quien sistematizó un informe de más de setenta casos de atención directa, doce consultorías, dos protocolos y cinco guías sobre derechos y deberes de la mencionada Clínica. Bariffi (2013), en Práctica clínica y litigación estratégica en discapacidad y derechos humanos, se refirió a varios ejercicios de trabajo clínico, tanto teórico como práctico, luego de la entrada en vigor de la Convención sobre los Derechos de las Personas con Discapacidad; Torres (2013), en "La enseñanza clínica del derecho: una forma de educación para el cambio social", se centró en el Grupo de Acciones Públicas de la Universidad del Rosario, indagando cómo el método clínico ha servido para establecer escenarios de formación práctico-académica y ha favorecido la atención de las necesidades legales de comunidades vulnerables.
Por su parte, García et al. (2014), en "Clínica jurídica, una forma de aprendizaje y servicio para la protección de derechos humanos", recordaron la puesta en marcha de la Clínica de la Universidad de Valladolid, cuyo empeño se afincó en la formación de juristas socialmente comprometidos. De Prada et al. (2014), en "La clínica jurídica de Villanueva. Función social y pedagógica del aprendizaje del Derecho", discutieron alrededor de sus herencias y del aprendizaje adquirido en términos éticos y jurídicos. Palma del Teso (2015), en "La clínica jurídica para la protección de la infancia y la adolescencia: experiencia educativa e investigadora desde el compromiso social, desarrollada en la Facultad de Derecho de la Universidad de Barcelona", ahondó en el factor social que debía envolver la práctica profesional de los estudiantes.

Adicionalmente, Pabón et al. (2015) relataron su experiencia de investigación y acción integral en desarrollo del "Proyecto de atención a estudiantes víctimas de matoneo por razones de orientación sexual", en un artículo con el mismo nombre. Vásquez-Sánchez (2016), en "La enseñanza clínica del derecho a partir de la experiencia de la Clínica Jurídica de Interés Público Unaula", extrajo los retos y las recompensas tras la ejecución de la propuesta de investigación "Indisciplinar el derecho". Pabón et al. (2016), en "La clínica jurídica como estrategia para fortalecer las competencias ciudadanas: una apuesta por la convivencia pacífica", disertaron en torno a los resultados del plan de atención y prevención del acoso escolar, realizado por la Clínica Jurídica de la Unab.

A su vez, Alonso (2016) indagó si las actividades clínicas habían superado el hecho de que las instituciones no estuvieran educando integralmente, al menos, en cuanto a lo emocional, social e intelectual, por medio de "La propuesta pedagógica de la Clínica Jurídica Internacional Penal y Humanitario de la Universidad del Rosario". Téllez et al. (2016), en "Aplicación de la enseñanza clínica del derecho a casos de violencia intrafamiliar", describieron el enfoque diferencial, transversal e interdisciplinario que ha tenido la Clínica de Violencia Intrafamiliar y Género de la Universidad del Rosario. Cosentino (2016) narró las enseñanzas pedagógicas y humanistas de la Clínica de la 
Universidad Nacional de La Plata, con respecto a cómo ha cambiado la vida de los estudiantes y de la población intervenida, en "La clínica jurídica de derecho ambiental: humanismo, reflexión y aprendizaje cooperativo para la evolución como personas y abogados".

León et al. (2016), en "Del método de enseñanza clínico hacia una pedagogía clínica en la enseñanza del derecho", se refirieron al origen y repercusión social conseguida por la Universidad de Ibagué a través de un estilo educativo no convencional. Pabón y Pinzón (2017), en "La experiencia de la Clínica Jurídica de Derechos Humanos e Interés Público", recuperaron un ejercicio de atención a menores con discapacidad, exponiendo cómo su intervención había servido para visibilizar una situación de quebrantamiento de derechos, algunas deficiencias en la gestión pública y las muchas ventajas de la formación clínica.

En cuarto lugar, enfatizando en las virtudes de la estrategia clínica, en un capítulo del libro Litigio y políticas públicas en derechos humanos, Puga (2002) analizó los desafíos de la implementación de método en la educación superior de Argentina, como correlato de un nuevo paradigma que, en los países sudamericanos, hasta la fecha de su publicación, no había tenido impacto. Villarreal y Courtis (2006), en La enseñanza clínica del derecho: una alternativa a los métodos tradicionales de formación de abogados, reunieron una serie de trabajos de expertos en el tema clínico, con la intención de promover dicha técnica en América Latina.

Castro-Buitrago (2006b), en "La enseñanza clínica. Un paso hacia la calidad", demostró la pertinencia de la metodología para satisfacer los requisitos de calidad en el sistema de educación superior colombiano, conforme con lo fijado por el Consejo Nacional de Acreditación (cNA); Cavallaro y Elizondo (2011), observaron las tendencias en la práctica del derecho internacional de los derechos humanos, rescatando la expansión y el espíritu innovador y social de que han traído las clínicas, en “¿Cómo establecer una clínica de derechos humanos?", Mezuera-Ayala (2012) insistió en las ventajas que conlleva una iniciativa de litigio estratégico, conciliación y trabajo interdisciplinario, que combina lo individual y lo colectivo, lo teórico y lo práctico, y lo ético y lo jurídico, en "Las clínicas jurídicas de interés público como escenarios de flexibilidad académica".

Bregaglio et al. (2013), bajo la premisa de que la educación clínica ha sido un motor para la formación integral, capaz de comprometer curricularmente contenidos en pro de la inclusión social, publicaron el Manual para el estudiante de la clínica jurídica en discapacidad y derechos humanos de la PUCP. Torres y Londoño (2014), en "Herramientas para la protección del interés público en América Latina", describieron los desafíos y presupuestos que han marcado la pauta del quehacer clínico en las universidades; y cómo se han orientado los esfuerzos hacia el diseño de instrumentos pedagógicos cada vez más vivos y activos. García (2014) ofreció algunas sugerencias para la incorporación clínica en la educación de profesionales y de ciudadanos, en "La integración de la educación jurídica clínica en el proceso formativo de los juristas".

Blázquez-Martín et al. (2014), en la Guía sobre cómo crear, organizar, gestionar y conducir una clínica jurídica en una facultad de Derecho, dieron respuesta a la solicitud del Colegio de Abogados de Madrid, resaltando la importancia de un aprendizaje protagónico y una cultura sobre los valores humanos y la conciencia social. Palacios (2014), en Re-intencionar las clínicas jurídicas: experiencia en la clínica jurídica de consulta previa, afirmó que las universidades debían propender por un aprendizaje interdisciplinar y, con especial afinidad, por la sociedad. Murcia y Londoño (2015) reconocieron el valor de las clínicas en materia de innovación y mediación pedagógica, y cómo han logrado fijarse en los estudiantes como eje del proceso, en "Avances en los lineamientos para la construcción de un modelo de educación legal clínica en América Latina".

Londoño-Toro (2015), en Educación legal clínica $y$ litigio estratégico en Iberoamérica, aclaró que este método puede ser observado como una respuesta complementaria para la reforma de la educación jurídica, evidenciando cómo, aparte de ser congruente con los planteamientos de Bolonia y reunir los elementos de la teoría clásica del derecho, del realismo jurídico y de la teoría crítica, se enfoca en los estudiantes como centro de transformación de 
la docencia, la investigación y la extensión. Torres et al. (2015), en El interés público en América Latina, reflexionaron alrededor del trabajo pro bono, de la educación legal clínica y del litigio estratégico como medios para la defensa del interés público y la transformación social. Santana et al. (2016), en "Clínica jurídica: potencialidades para la enseñanza y el aprendizaje de las ciencias penales y criminológicas", recalcaron su carácter flexible, participativo y alternativo, así como su capacidad para originar cambios y transformaciones.

A su turno, Gascón (2016), en "La evolución de la enseñanza clínica en las universidades españolas: oportunidades y desafíos de la litigación estratégica", insistió en la complementariedad, función social, servicio púbico, conjunción teórico-práctica y transferencia de saberes disciplinares y populares, que trae consigo la metodología clínica. Valenzuela (2016), en Transversalización del enfoque de derechos humanos en el área de enseñanza clínica del derecho, destacó las orientaciones técnicas y buenas prácticas de la Universidad Central de Chile, en cuanto a los requerimientos de capital humano, adecuación logística y planeación metodológica que entrañan el modelo. Por último, Londoño-Toro (2016), en "Los cambios que requieren las clínicas jurídicas iberoamericanas: estudio de caso en seis países de la región”, recogió la perspectiva de distintos profesores y estudiantes acerca de los retos y las necesidades de las clínicas respecto de la formación jurídica, y evaluó su aporte en las universidades.

En síntesis, la evolución del discurso en torno a la educación clínica ha acentuado la urgencia de resignificar la formación profesional en derecho. Pero sus avances han demandado perseverancia y el esfuerzo mancomunado de los estamentos universitarios. Aunque es sabido que la técnica ha logrado resultados trascendentales en términos de calidad, integralidad, interdisciplinariedad y responsabilidad; su implementación ha exigido compromisos extraordinarios que van desde la creación de alianzas y asociación a redes, pasando por la disposición de una agenda continua de capacitaciones, la expansión curricular y extracurricular, la participación en labores académico-investigativas y la conducción de empresas solidarias, hasta la consolidación de un presupuesto específico, destinado a la flexibilización y profundización educativa.

\section{La transversalización de la educación clínica jurídica}

En el plan conducido a instancias de la Unab, la pretensión fue aprovechar la existencia y el reconocimiento de la clínica existente, para extender sus operaciones más allá del centro de práctica profesional: consultorio jurídico; transversalizando sus premisas en los cursos regulares del pénsum. Dicho compromiso encarnó, por una parte, la oportunidad de enmendar la ausencia o insuficiencia de los saberes prácticos, la pérdida de confianza y autonomía, y de aptitudes para hacer del aprendizaje un ejercicio de vida y no solo de promoción o graduación; y la desatención de las habilidades para relacionarse con los otros, trabajar colectivamente y comunicarse de modo efectivo (Cajide et al., 2002; Ginés, 2004; García y Ginés, 2000); por otra, la invitación a una educación emocional en derechos humanos (Rorty, 2000), es indispensable para la edificación de valores democráticos y no-violentos en comunidad.

Haber enfocado de esta manera el modelo clínico originó un marco virtuoso para la formación de buenos profesionales y ciudadanos. Las actividades fueron guiadas no por descriptores o por las unidades temáticas comunes en los libros de historia del derecho ${ }^{10}$, sino por preguntas abiertas carentes de respuestas absolutas o de conceptos unívocos, cuya redacción buscó involucrarlos en la construcción del conocimiento. A saber,

10 En la mayoría de los casos, es posible predecir las tablas de contenido de los manuales de historia del derecho en Occidente; al menos en los países de la civil law. No suelen obviarse, por ejemplo, los siguientes apartados o títulos: derecho romano (preclásico, clásico y posclásico), derecho bizantino, vulgarización del derecho romano, escuelas del derecho (glosadores, posglosadores, jus-humanistas, jus-racionalistas, escuela histórica y pandectistas), derecho indiano y codificaciones del siglo XIX. 
¿de qué hablamos cuando nos referimos al derecho?, ¿cómo llegamos a la comprensión del derecho como un sistema normativo?, ¿cómo se ha construido, a través del tiempo y el espacio, nuestra tradición jurídica?, ¿cuáles son sus sentidos y funciones?, ¿quién han estado a cargo de su materialización?, ¿quiénes han sido, somos y seremos los llamados sujetos de derecho?, ¿qué significa ser sujeto de derechos y cuáles son los llamados derechos en cabeza de cada individuo-entidad?, ¿cómo han evolucionado los derechos y la búsqueda de potestades, libertades y demandas?, ¿para qué existen los derechos y cómo se han ido descubriendo o afianzando a lo largo de la historia?, y ¿cómo se cumple lo aprendido en nuestro entorno y de qué depende su aseguramiento?

En este sentido, la transversalización clínica supuso priorizar la formación por competencias ${ }^{11}$, partiendo del reconocimiento de cada persona y de la importancia de sus voces, practicando una convivencia inclusiva ${ }^{12}$, observando los desacuerdos como oportunidades de deliberación y de crecimiento, y aplicando metodologías activas de

11 De acuerdo con el "Proyecto educativo institucional” de la Unab (2012), la formación por competencias es un enfoque educativo multidimensional, centrado en el "desarrollo de las capacidades y habilidades requeridas por una persona, para desenvolverse en diferentes escenarios" (p. 31).

12 En palabras de Furman (2003, p. 4), la inclusión educativa se traduce en la promoción de "relaciones sustentadas en la comunicación, el diálogo y la colaboración" (11). Esto es, "favorecer procesos de conocer, comprender y valorar a los individuos, sus culturas de casa y las comunidades en que viven; [favorecer] procesos de participación plena, de indagación abierta y constructiva [...]; crear espacios para el diálogo y la deliberación, para hablar y pensar juntos; y [favorecer] procesos comunitarios para trabajar hacia el bien común, tratando de vincular ideas con proyectos y acciones específicas" (citado por Fierro (2011, p. 12). aprendizaje ${ }^{13}$. Tras la etapa de fundamentación, se exploraron y escogieron situaciones-problemas de base cotidiana. Los equipos, ya organizados, se dieron a la tarea de idear un plan de acción, de cara a cada conflicto y fueron perfeccionándolo y ejecutándolo con el paso de las semanas.

Puntualmente se conformaron once equipos. Uno de ellos se enfocó en cambiar el discurso sobre las personas habitantes de la calle, procurando subsanar algunas de sus necesidades cardinales, impulsando la responsabilidad social corporativa, e implementando rutas de gobernanza, mediante la coordinación de un evento titulado "Calles de solidaridad". Este evento reunió personas naturales, empresas de la región y entidades estatales. Otros dos grupos, habiendo observado dificultades y oportunidades de mejora en el acceso a la infraestructura y los servicios públicos de las personas con discapacidad, aprovecharon un espacio institucional de bienestar universitario llamado "Feria de la salud", para crear dos stands y educar de manera sensible a los estudiantes de pregrado, bajo el argumento de que no es la población con discapacidad quien debe adaptarse al mundo, sino que es la sociedad la que debe implementar políticas y prácticas de inclusión; un equipo se puso en contacto con algunos colegios públicos y privados para efectuar junto a los estudiantes de último año la dinámica "Detectatrata", incluida en la Guía para talleres de multiplicación sobre la trata de personas de la Organización Internacional para las Migraciones (огм, 2010), con destino a advertir la latencia de dicha conducta punible e identificar los elementos en común y diferencias entre la trata de personas y el tráfico ilícito de migrantes.

Con respecto a los patrones de discriminación por razones de género, se asociaron dos grupos para recrear un "Laboratorio para la inclusión", donde realizaron un concurso para inventar y

13 Según Fernández-March (2006), las metodologías activas de aprendizaje abarcan los procedimientos y las técnicas, donde la responsabilidad educativa depende directamente de la actividad y de la implicación y compromiso de los estudiantes, superando con ello las dinámicas pasivas o meramente informativas. 
emprender soluciones frente a la estigmatización, la violencia (directa, estructural y cultural) y la limitación de las oportunidades laborales y generación de ingresos por parte de las personas trans. Un equipo más se introdujo en otra manifestación de la desigualdad de género, pero en el ámbito rural, poniendo en práctica en veredas de los municipios de Lebrija, Los Santos y Piedecuesta, uno de los talleres del Manual y caja de herramientas de perspectiva de género de la Organización de las Naciones Unidas para la Alimentación y la Agricultura (FAO, 2018).

Con ello, se buscaba, por un lado, indagar en las diferencias reales y aparentes entre hombres y mujeres y, por otro, analizar cómo muchas de éstas han derivado en fenómenos de discriminación. Cuatro grupos se unieron para dar continuidad al "Cine-discusión", programando una cartelera mensual temática e instalando un escenario para la contextualización y deliberación interdisciplinar acerca de la realidad de la población migrante en la ciudad. Sobre el mismo tema, el último equipo se centró en las amenazas recibidas por los migrantes venezolanos, así como en las barreras que ciñen sus garantías laborales, diseñando un juego de roles, para llamar la atención con respecto a conductas xenófobas, intentando transformarlas en iniciativas para el restablecimiento de sus derechos.

Al final, (1) se abrió un camino para la transformación pacífica de diversas expresiones de violencia, estereotipación, discriminación, incitación al odio o mercantilización de la vida y la integridad; (2) se impuso la solidaridad, la educación, el fortalecimiento y la legitimidad de las instituciones, la identidad, la autonomía y la equidad, como elementos para la fractura de una lógica histórica de exclusión; (3) se robusteció la confianza en el mundo escolar; (4) se reclamó la sinergia de los agentes universitarios y de terceros a los protocolos de trabajo; y (5) se concedió un valor agregado al saber profesional, inclusive más complejo que la razón misma de la clase; dejando huellas en términos de conocimiento y de poder.

Adicionalmente, el plan de transversalización denotó una verdadera práctica educativa para la paz, entendida como un ejercicio inacabado de orden social (Muñoz, 1998), dirigido a alcanzar consensos en torno a convicciones que incluyeron una pluralidad de proyectos vitales, lenguajes, estilos de vida, ideologías, modelos sociopolíticos y sistemas económicos. Tales consensos inspiraron pautas de conducta ética y moral, y la comprensión y empatía necesarias para legitimar una existencia y convivencia dignas (Fisas, 2011).

\section{Conclusiones}

En palabras de Frank (1933), el derecho más que una ciencia es un arte y solo un pequeño fragmento de cualquier arte puede aprenderse con un modelo meramente doctrinal. La educación clínica, con el propósito de corregir este hecho, como han señalado Dinerstein (1992) y Clark (1993), consigue introducir a los estudiantes en problemas reales, exigiéndoles posturas cognitivas, analíticas, interpretativas, creativas, éticas e interactivas, para arribar a soluciones o respuestas igual de consistentes. Esta forma de educación se enfoca en cuestiones sociales y de interés público, amparando los derechos humanos de personas y sectores vulnerables; acoge el aprendizaje de competencias no solo disciplinares sino también transversales y de habilidades blandas, en función del trabajo cooperativo; y comporta una evaluación permanente.

Tras la experiencia en la Unab, pudo comprobarse, igualmente, que las clínicas configuran una ruta para la construcción de paz desde las aulas universitarias. En relación con este argumento, siguiendo a Hernández (2009), los emprendimientos ciudadanos y, entre estos, los que residen en el sistema educativo representan verdaderos espacios para edificar una cultura de paz. Sin duda, son capaces de crear realidades para visibilizar problemas o individuos, comunidades y pueblos, dentro de un territorio, a través de iniciativas concretas, cuya importancia está dada por el grado de profundización de las cargas axiológicas.

En lugar de concebirse este ejercicio como un proyecto estrictamente curricular y una actividad especializada en el mundo del derecho, probablemente su mayor fuerza radicó en el carácter práctico y significativo del aprendizaje que, si bien surgió 
en el curso de diacrónica jurídica, en ningún momento se limitó a la tarea de un par de semanas ni a una nota ni a una calificación. Con todo, para algunos estudiantes fue difícil romper los hábitos adquiridos durante años de formación, especialmente los relacionados con la exhaustividad de contenidos, el protagonismo exclusivo y excluyente de los profesores durante las clases y una evaluación concentrada en la memoria y el recuento de datos. No obstante, el ritmo clínico dispuesto pudo despertar, multiplicar y potenciar fibras en torno a una expresión distinta de educación, que acabó agudizando su responsabilidad social y no solo escolar.

La metodología clínica materializó una comunidad de indagación ${ }^{14}$ donde ni la ausencia de energía ni la indiferencia restringieron las posibilidades de empoderamiento. El aula ofreció una atmósfera libre de orgullos, haciendo que sus participantes apostaran por una alternativa de conocimiento y servicio pluriuniversitario (de Sousa, 2005), sin escindir los factores morales y la proximidad del derecho con el concepto de justicia. Ahora bien, la infraestructura de las clínicas es muy exigente, pues precisa de ambientes y recursos especiales para llevar a cabo reuniones que favorezcan la creatividad y eliminen las distracciones originadas por las jerarquías. De igual forma, demandan un capital humano calificado y, teniendo en cuenta que constituyen un ejemplo de emprendimiento social, cuya renta no suele estar dada (directamente) en términos de ganancias económicas, es indefectible la adhesión desde el plano institucional y organizacional, para estar seguros de su sostenibilidad, continuidad y progresividad (Londoño-Toro, 2016).

En resumen, la transversalización del método se ubicó en la esfera de lo factible y lo efectivo, que

14 Las comunidades de indagación y aprendizaje buscan que los sujetos intervinientes sean "parte de experiencias sociales que posibiliten el desarrollo autónomo, la convivencia cooperativa y el diálogo de saberes” (Messina, s.f., p. 8). En este sentido, el método de clínica jurídica buscó incentivar un aprendizaje crítico, significativo, integral, experiencial y preocupado por la emancipación y el empoderamiento poblacional. emergió como una política de educación jurídica, democracia y paz en la que los estudiantes pudieron verse como sujetos de derechos, y terciaron en el proceso pedagógico, promoviendo su desarrollo integral y el de las poblaciones alcanzadas. Al momento de ponderar la intervención en el curso y los planes de acción adelantados, cotejando las preferencias de los estudiantes respecto de otros cursos y las expectativas frente a los resultados, fue posible notar también la conveniencia de su implementación ${ }^{15}$.

Así, a pesar de los retos que la innovación y los cambios trajeron consigo, se hizo énfasis en la construcción de valor a partir del trabajo en equipo, comprendido no como la posibilidad de fraccionar tareas, sino como una dinámica cooperativa-colaborativa en la que se compartieron voluntades y esfuerzos, y se socializaron saberes para gestar nuevas competencias. Se demostró la concepción del conocimiento no como algo inmutable, sino como un producto significativo con soporte teórico y empírico. Se subrayó el giro en el sistema de evaluaciones $y$, aunque inicialmente suscitó incertidumbre, los estudiantes dejaron de sentirse atemorizados por lo que percibían como un juicio unilateral.

Adicionalmente, se advirtió la mayor dedicación de tiempo que exigió la clase, sugiriendo con ello una planificación mancomunada entre profesores para una completa descentralización del modelo, a fin de que cada curso no denote una multiplicación de actividades, sino una interrelación de metas. Y, por último, se observó un nivel elevado en la calidad, mediante el reconocimiento y comprensión del sustrato histórico acerca de instituciones jurídicas y fenómenos políticos, sociales, económicos y culturales que, más tarde, condujeron al planteamiento y ejecución de soluciones

15 La voz de los estudiantes se expresó en este sentido a través de dos herramientas: (1) en la encuesta institucional de evaluación docente y (2) al término del periodo académico en un último grupo de discusión con once estudiantes, a razón de un miembro por cada equipo, asegurando heterogeneidad en cuanto a temas y experiencias, $\mathrm{y}$ homogeneidad en virtud del nivel educativo y pertenencia a la clase de Diacrónica Jurídica. 
razonadas y razonables, en las que se verificaron actos comunicativos asertivos y coherentes.

\section{Referencias}

Abramovich, V. (1999). La enseñanza del derecho en las clínicas legales de interés público. Materiales para una agenda temática. Cuadernos de Análisis Jurídico, 9, 6193.

Alonso, H. (2016). La propuesta pedagógica de la clínica jurídica internacional: la formación del ser humano como un todo. En H. Hola solo (Ed.), Derecho internacional penal y humanitario. Estudios de la Clínica Jurídica Internacional de la Universidad del Rosario (Colombia) y del Instituto Iberoamericano de la Haya para la Paz, los Derechos Humanos y la Justicia Internacional (Holanda) en su $V$ Aniversario (pp. 31-42). Tirant lo Blanch.

Atienza, M. (1997). Los límites de la interpretación constitucional. De nuevo sobre los casos trágicos. Isonomía: Revista de Teoría y Filosofía del Derecho, 6, 7-30.

Atienza, M. y Ruiz, J. (1991). Sobre reglas y principios. Revista DOXA, 10, 101-120. https://doi.org/10.14198/ DOXA1991.10.04

Bariffi, F. (2013). Práctica clínica y litigación estratégica en discapacidad y derechos humanos. Algunas experiencias de Iberoamérica. Dykinson.

Bayuelo, P. (2015). La educación y el derecho en torno a un nuevo paradigma transformador. Justicia $27,167-84$.

Blázquez-Martín, D. (2006a). Apuntes acerca de la educación jurídica clínica. Universitas, 3, 43-60.

Blázquez-Martín, D. (2006b). La educación jurídica clínica en el contexto del 'Proceso De Bologna'. Su aplicabilidad en España. Revista Opinión Jurídica, 5(10), 161-79.

Blázquez-Martín, D, Cuenca-Gómez, P. e Iglesias-Garzón, A. (s.f.). Guía sobre cómo crear, organizar, gestionar y conducir una clínica jurídica en una facultad de derecho. Icam.

Borràs, C. (2012). La clínica jurídica de la Universitat de València: el compromís social de l'aprenentatge participatiu. Futura 24, 35-37.

Borrero, C. (2014). Explorando la sociología jurídica. Una propuesta de cátedra participativa. Universidad Nacional.

Bregaglio, R., Constantino, R. y Ocampo, D. (2013). Manual para el estudiante de la clínica jurídica en discapacidad y derechos humanos de la PUCP. Instituto de Democracia y Derechos Humanos.
Cajide, J., Porto, A., Abeal, C., Barreiro, F., Zamora, E., Expósito, A., y Mosteiro, J. (2002). Competencias adquiridas en la universidad y habilidades requeridas por los empresarios. Revista de Investigación Educativa 20(2), 449-67.

Carrillo, A. y Espejo, N. (2013). Re-imaginando la clínica jurídica de derechos humanos. Academia 11(22), 1553.

Castro-Buitrago, E. (2006a). Desarrollo de un método de selección de casos de interés público. Experiencia de la clínica jurídica de interés público de la Universidad de Medellín. En O. C. Restrepo, Investigación jurídica y sociojurídica en Colombia. Resultados y avances en investigación (pp. 71-82). Universidad de Medellín.

Castro-Buitrago, E. (2006b). La enseñanza clínica. Un paso hacia la calidad. Opinión Jurídica, 5(9), 175-86.

Castro-Buitrago, E. (2015). Un modelo de estrategia didáctica para la enseñanza clínica del derecho con énfasis en investigación. Revista Indisciplinas, 1(1), 69-98.

Buitrago, E. J. C. (2004). Perspectivas de la enseñanza clínica del derecho en Colombia. Opinión Jurídica, 3(5), 161-168.

Cavallaro, J. y Elizondo, F. (2011). Cómo establecer una clínica de derechos humanos? Lecciones de los prejuicios y errores colectivos en las Américas. Justicia Constitucional, 6, 124-40.

Clark, E. (1993). Legal education and professional development -an educational continuum, report of the task force on law schools and the profession. Narrowing the gap (Illinois: American Bar Association 1992). Legal Education Review, 4(1). http://www.austlii.edu.au/au/ journals/LegEdRev/1993/9.html

Congreso de Colombia (1992, 28 de diciembre). Por la cual se organiza el servicio público de la Educación Superior [Ley 30 de 1992]. Diario Oficial, No. 40700. http://www.legal.unal.edu.co/rlunal/home/doc.jsp?d_ $\mathrm{i}=34632$

Correa, L. y Vásquez J. (2013). La enseñanza clínica del derecho: transformando la forma de enseñar y ejercer el derecho. En La Enseñanza Clínica del Derecho: 105-26.

Cosentino, G. M. (2016). La clínica jurídica de derecho ambiental: humanismo, reflexión y el aprendizaje cooperativo para la evolución como personas y como abogados. I Jornadas sobre las Prácticas Docentes en la Universidad Pública. Transformaciones actuales y desafíos para los procesos de formación (La Plata, 2016). http:// sedici.unlp.edu.ar/handle/10915/61660

Courtis, Ch. (2007). La educación clínica como práctica transformadora. En M. Villarreal y Ch. Courtis (Coord.), Enseñanza clínica del derecho. Una alterna- 
tiva a los métodos tradicionales de formación de abogados (pp. 9-24). Clip-Itam.

De Prada Rodríguez, M., Callejo, S., \& López, P. (2014). La clínica jurídica Villanueva: Función social y pedagógica del aprendizaje del Derecho. Reduca (Derecho), 5(1), Article 1. http://revistareduca.es/index.php/reduca-derecho/article/view/1851

De Sousa, B. (2004). Democratizar la democracia. Los cambios de la democracia participativa. Fondo de Cultura Económica.

De Sousa, B. (2010). La universidad del siglo xxı. Para Una reforma democrática y emancipatoria de la universidad. Cides-Umsa.

Dinerstein, R. (1992). Report of the Committee on the Future of the in-House Clinic, August 1990, Revised October 1991. Journal of Legal Education, 42(4): 508-74.

Estrada, S. (2015). De las aulas a la calle: un paso más de la racionalidad teórica hacia la racionalidad práctica. Aportes y retos de la clínica jurídica a la enseñanza de la teórica general del derecho. Revista Indisciplinas, 1(1): 47-68.

Fernández-March, A. (2006). Metodologías activas para la formación de competencias. Educatio Siglo xxı, 24, 35-56.

Fierro-Evans, M. (2013). Convivencia inclusiva y democrática. Una perspectiva para gestionar la seguridad escolar. Sinéctica 40, 01-18.

Figueroa-Camacho, T. (2011). La enseñanza del derecho: más allá del formalismo (Tesis de Maestría, Universidad Nacional de Colombia). Repositorio Institucional Unal: https://repositorio.unal.edu.co/handle/ unal/11257

Fisas, V. (2011). Educar para una cultura de paz. Quaderns de construcció de pau, 20, 17-31.

Frank, J. (1933). Why not a clinical lawyer-school? University of Pennsylvania Law Review and American Law Register, 81(8), 907-23.

García (2014). La integración de la educación jurídica clínica en el proceso formativo de los juristas. Redu. Revista de Docencia Universitaria, 12(3), 153-175. https:// doi.org/10.4995/redu.2014.5495

García, J. (2015). La evolución de la educación jurídica clínica en España. Revista de Educación y Derecho. Education and Law Review, 11(9), 1-6.

García, J. y Ginés, J. (2000). El mercado laboral de los titulados superiores en Europa y en España. Papeles de Economía Española, 86, 111-27.

García, J., Fanego, C. A., Fernández, B. V., Sancho, M. de H., Luelmo, A. D., Santos, F. J., Martín, C. G., Tomillo,
M. G., Pérez, C. V., Pérez, E. M., Añón, J. G., Corella, A. S., Pérez, L. S., Ribas, A. F. y Ferrer, V. R. (2014). Clínica jurídica, una forma de aprendizaje-servicio para la protección de derechos humanos. Reduca (Derecho), 5(1), 110-122. http://revistareduca.es/index.php/reduca-derecho/article/view/1841

Gascón, A. (2016). La evolución de la enseñanza jurídica clínica en las universidades españolas: oportunidades y desafíos de la litigación estratégica en las clínicas de derechos humanos. Revista de Educación y Derecho, 14, 1-15.

Ginés, J. (2004). La necesidad del cambio educativo para la sociedad del conocimiento. Revista Iberoamericana de Educación, 35(1), 13-37.

Gómez-Araújo, L. (2011). ¿Existe crisis en la enseñanza del derecho? Revista de Derecho, 13(13), vii-xii.

González-Morales, F. (2004). El trabajo clínico en materia de derechos humanos e interés público en América Latina. Universidad Deusto.

Hernández, E. (2008). La paz imaginada por quienes la construyen: iniciativas civiles de paz de base social identifican sus sueños de paz. Reflexión Política 10(19), 134-147.

Hernández, E. (2009). Paces desde abajo en Colombia. Reflexión Política 11(2), 176-186.

Hurtado-Castrillón, L. F. (2011). Clínica jurídica en acciones de interés público en materia de discapacidad para el eje cafetero. Memorando de Derecho 2(2): 51-62.

Lariguet, G. (2004). Conflictos trágicos y derecho. Posibles desafíos. Cuadernos de Filosofía del Derecho, 27, 317348. https://doi.org/10.14198/DOXA2004.27.11

León, A., Pinzón, I. y Mejía, O. (2016). Del método de enseñanza clínico a una pedagogía clínica en la enseñanza del derecho. Una experiencia desde la clínica de interés público de la Universidad de Ibagué. Revista indisciplinas, 2(3), 97-126.

Londoño-Toro, B. (2015). Educación legal clínica y litigio estratégico en Iberoamérica. Universidad del Rosario.

Londoño-Toro, B. (2016). Los cambios que requieren las clínicas jurídicas iberoamericanas. Estudio de caso en seis países de la región. Boletín Mexicano de Derecho Comparado, 49(146), 119-48.

MacLeod, G. (1963). Creative problem-solving... for lawyers? Journal of Legal Education, 16(2), 198-202.

Martocci, J. (2017). Clínica jurídica en derechos humanos, otra pedagogía. Revista de Interés Público (Redip), 1(1), 23-36. 
Merla, A. y Yáñez, C. (2016). El aula invertida como estrategia para la mejora del rendimiento académico. Revista Mexicana de Bachillerato a Distancia 8(16), 68-78.

Merlín, R. (2016). De la enseñanza tradicional del Derecho a la enseñanza creativa y constructiva del derecho. En Cáceres, E. (Coord.), Pasos hacia una revolución en la enseñanza del derecho en el sistema romano-germánico (Tomo II. pp. 477-486). Instituto de Investigaciones Jurídicas, Unam.

Messina, G. (s.f.). Unidad 4 - Anexo 2. Notas acerca de la comunidad de aprendizaje. Material didáctico del curso Metodología para la ejecución y la evaluación de proyectos de EDH II. Crefal.

Mezuera-Ayala (2012) Las clínicas jurídicas de interés público como escenarios de flexibilidad académica. $M e-$ morando de Derecho 3(3), 123-132.

Molina-Saldarriaga, C. (2012). La enseñanza clínica del derecho. Presupuestos metodológicos y teóricos para la inclusión de la interdisciplinariedad en la formación jurídica. Ratio Juris 7(15), 81-104.

Muñoz, F. (1998). La paz imperfecta. Apuntes para la reconstrucción del pensamiento pacifista. Papeles de Cuestiones Internacionales 65, 11-14.

Murcia, P. y Londoño, B. (2015). Avances en los lineamientos para la construcción de un modelo de educación legal clínica en América Lanita. En El interés público en América Latina: reflexiones desde la educación legal clínica y el trabajo pro bono (pp. 34-75). Universidad del Rosario.

Organización de las Naciones Unidas para la Alimentación y la Agricultura -FAo. (2018). Manual y caja de herramientas de perspectiva de género. Autor.

Organización Internacional para las Migraciones -oIM (2010). Un trato contra la trata: Guía para talleres de multiplicación sobre la trata de personas. Autor.

Pabón, A. (2015). Proyecto de atención a estudiantes víctimas de matoneo por razones de orientación sexual: reflexión de su formulación al interior de la línea de género y derecho de la clínica jurídica de la Unab. Revista Indisciplinas 1(2), 27-43.

Pabón, A., Aguirre, J. y Cáceres, P. (2016). La clínica jurídica como estrategia para fortalecer las competencias ciudadanas: una apuesta por la convivencia pacífica. Ratio Juris, 11(23), 27-46.

Pabón, A. y Pinzón, D. (2017). La experiencia de la clínica jurídica de derechos humanos e interés público. Advocatus, 14(27), 17-34. https://doi.org/10.18041/01240102/advocatus.27.914
Palacios, Y. 2014. Re-intencionar las clínicas jurídicas: mi experiencia en la clínica jurídica de consulta previa. Opinión Jurídica, 13(26), 189-190.

Palma del Teso, Á. (2015). La clínica jurídica para la protección de la infancia y la adolescencia. Experiencia educativa e investigadora desde el compromiso social, desarrollada en la facultad de derecho de la Universidad De Barcelona. Revista de Educación y Derecho, 11, 1-15. https://doi.org/10.1344/re\&d.v0i11.12000

Puga, M. (2002). Los desafíos de las clínicas jurídicas en Argentina. En F. González (Ed.), Litigio y políticas públicas en derechos humanos (pp. 41-93). Universidad Diego Portales.

Rancière, J. (1996). El desacuerdo: política y filosofía. Nueva Visión.

Raudsepp, E. (1963). Managing creative scientists and engineers. Macmillan.

Red-DESC (1999). Observación general no. 13. El derecho a la educación (artículo 13 del Pacto Internacional de Derechos Económicos, Sociales y Culturales). https:// www.escr-net.org/es/recursos/observacion-general-no-13-derecho-educacion-articulo-13

Rodríguez, G. (2007). Prólogo. En M. Villarreal y Ch. Courtis (Coord.), Enseñanza clínica del derecho. Una alternativa a los métodos tradicionales de formación de abogados (pp. 5-7). México: Clip-Itam.

Rorty, R. (2000). Derechos humanos, racionalidad y sentimentalismo. Verdad y Progreso, 1, 219-242.

Santana, L., Pereda, A. y Mirabal, M. (2016). Clínica Jurídica: potencialidades del método para el proceso enseñanza-aprendizaje de las ciencias penales y criminológicas. Revista de Ciencias Médicas de Pinar del Río, 20(4), 142-51.

Taelli-Gómez, F. (2014). Paradigmas emergentes y crisis en la educación del Derecho. Revista Pedagogía Universitaria y Didáctica del Derecho, 1(1), 58-71. https://doi. org/10.5354/0719-5885.2014.35963

Téllez, M., Mancera, N. y Vélez, P. (2016). Aplicación de la enseñanza clínica del derecho en casos de violencia intrafamiliar. Revista Indisciplinas, 2(3), 53-72.

Torres, M. (2013). La enseñanza clínica del derecho: una forma de educación para el cambio social la experiencia del grupo de acciones públicas de la Universidad del Rosario. Revista de la Facultad de Derecho y Ciencias Políticas, 43(119).

Torres, M., Iregui, P. y Senior, S. (2015). El interés público en América Latina. Reflexiones desde la educación legal clínica y el trabajo pro bono. Editorial Universidad del Rosario. 
Torres, M. y Londoño, B. (2014). Herramientas para la protección del interés público en América Latina: el diseño de un litigio de alto impacto desde la educación legal clínica. Teoría y derecho: revista de pensamiento jurídico 15: 92-107.

Unab. Junta Directiva. (2012). Proyecto educativo institucional. Autor.

Unab. Junta Directiva. (2014). Acuerdo 092 de 2014: Por medio de la cual se promulga el Reglamento de la Clínica Jurídica adscrita al Consultorio Jurídico de la Universidad Autónoma de Bucaramanga. Autor.

Valenzuela, M. (2016). Transversalización del enfoque de derechos humanos en el área de enseñanza clínica del derecho. En E. Cáceres (Coord.), Pasos hacia una revolución en la enseñanza del Derecho en el sistema romano germánico (pp. 359-372). Unam.

Valle-Acevedo, A. (2006). Cultura jurídica y enseñanza del derecho. ¿Creencias o competencias? Revista Escuela de Derecho, 7(7), 85-98.
Vásquez-Santa María, J. (2016). La enseñanza clínica del derecho a partir de la experiencia de la clínica jurídica de interés público Unaula. Revista Indisciplinas, 2(4), 121-55.

Vázquez-Sánchez, O. (2006). La argumentación jurídica en el Tribunal Constitucional español. Los casos fáciles, difíciles... trágicos. Revista Telemática de Filosofía del Derecho 9, 191-221.

Vázquez, R. (2006). Cómo se enseña el derecho. Ciencia: Revista de la Academia Mexicana de Ciencias, 57(2). https://www.revistaciencia.amc.edu.mx/images/revista/57_2/comoseensena_derecho.pdf

Villarreal, M. y Courtis, C. (2006). Enseñanza clínica del derecho. Una alternativa a los métodos tradicionales de formación de abogados. México: Clip-Itam. 